\title{
Unusual Synchronous Presentation of Maxillary Sinus Fibrosarcoma and Gemistocytic Astrocytoma with a Complication Called Leukocytoclastic Vasculitis: A Case Report
}

\author{
Bilge Cadir ${ }^{a}$ \\ Nermin Karahan ${ }^{b}$ \\ Serdar Nasir ${ }^{c}$ \\ M. Asim Aydinc \\ S. Suha Turkaslan ${ }^{d}$
}

\section{ABSTRACT}

Fibrosarcoma of the paranasal sinuses is extremely rare pathology and there is limited report in the literature. We report synchronous presentation of maxillary sinus fibrosarcoma and gemistocytic astrocytoma which is, to our knowledge, unique in the literature. Both tumors metastases to other organ rarely and the metastatic spread of gemistocytic astrocytoma to fibrosarcoma or vice versa have also not been reported in the literature yet. This report discusses the clinical course of the disease, outcome of the treatment approach and survival as well as an unusual occurrence of leukocytoclastic vasculitis during the course of radiotherapy in such unusual presentation. (Eur J Dent 2009;3:233239)

Key words: Fibrosarcoma; Gemistocytic astrocytoma; Synchronous cancer; Maxillary sinus; Leukocytoclastic vasculitis; Radiotherapy.

a Department of Oral and Maxillofacial Surgery, Faculty of Dentistry, Suleyman Demirel University, Isparta, Turkey.

b Department of Pathology, Faculty of Medicine, Suleyman Demirel University, Isparta, Turkey.

Department of Plastic and Reconstructive Surgery, Faculty of Medicine, Suleyman Demirel University, Isparta, Turkey.

d Department of Prosthetics, Faculty of Dentistry, Suleyman Demirel University, Isparta, Turkey.

- Corresponding author: Bilge Cadir

Suleyman Demirel Universitesi

Dis Hekimligi Fakultesi

Oral ve Maksillofasiyal Cerrahi A.D. 32260

Isparta, Turkey.

Phone : 00902462113289 Fax: 00902462370607

E-mail : bilge.cadirdyahoo.com

\section{INTRODUCTION}

Malignant tumors of the paranasal sinuses constitute $3 \%$ of all head and neck cancers. ${ }^{1}$ Among these, paranasal fibrosarcoma, which is a malignant tumor of the fibroblasts, is extremely rare and there is limited report in the literature. Paranasal sinus sarcomas constitute $7 \%$ of all head and neck sarcomas ${ }^{2}$ and lower than $1 \%$ of cancers in this region..$^{3,4}$ On the other hand, gemistocytic astrocytoma which originates from astrocytes is a histological variant of low-grade diffuse astrocytoma (WHO grade II), and has high frequency of rapid tumor recurrence and malignant progression. ${ }^{5,6}$ It grows slowly; however often behave aggressively because of the presence of 
gemistocytic cells which are neoplastic in nature. The incidence rate adjusted to the World Standard Population, per million populations per year, is $2.28 .{ }^{7}$ Considering the incidence of entire low-grade diffuse astrocytomas, it is also a rare condition. Local recurrences and distant metastases may occur in both fibrosarcoma and gemistocytic astrocytoma. However, there is no report in which the metastatic spread of gemistocytic astrocytoma to fibrosarcoma or vice versa in the literature. An occurrence of multiple primary cancers with either gemistocytic astrocytoma or fibrosarcoma is also extremely rare. We reported this case since, to our knowledge, the patient was unique in whom maxillary sinus fibrosarcoma and gemistocytic astrocytoma of brain developed synchronously as separate primary tumors. Furthermore, although there have been several previous reports on sarcomas of the head and neck, only few of them report sarcoma of the oral and maxillofacial region. ${ }^{8-11}$ The limited number of patients reported in these papers demonstrates the rarity of sarcomas in the oral and maxillofacial region (14 patients over 15 years, ${ }^{8} 25$ patients over 35 years, ${ }^{9} 10$ patient over 8 years, ${ }^{10}$ and 32 patients over 25 years ${ }^{11}$. As a consequence of their variety of the originating tissue and their rarity, there is need to discuss clinical and pathological features, to establish standard treatment regime, and to be able to estimate prognosis of sarcomas of the oral and maxillofacial region. Finally, the diagnosis of sinonasal malignancies is difficult. Since the early diagnosis is one the most important factor for favorable treatment outcome, to detect the possible early signs and symptoms of paranasal sinus cancer is essential. Therefore, the aim of this case report was to discuss the clinical course of the disease, outcome of the dual therapy with surgery and radiotherapy, unusual development of leukocytoclastic vasculitis during radiotherapy, and survival in such unusual presentation. Such data deserves to be recorded for the management protocols of prospective cases.

\section{CASE REPORT}

A 48-year-old male Turkish farmer was admitted to our clinic with complaints of slight pain, sense of swelling and discomfort in the left-check, pressure to left eye with two-month duration. Past medical history of the patient revealed an excision of a brain tumor and its radiotherapy a year ago. Histopathological report, sections (Figure 1a (100x Hematoxilen eosin (HE)); Figure 1b (200xHE)), and previous computerized tomography (CT) (Figure 2a) obtained from Antalya Government Hospital, where the brain tumor operation was performed, revealed that the tumor was a gemistocytic astrocytoma (Grade II - poorly differentiated mass). The histopathological sections (Figure 1a (100xHE); Figure 1b (200xHE)) were characterized with a predominance of gemistocytes, which are neoplastic cells. Voluminous, eosinophilic cytoplasm, eccentric nuclei, plump cell process and marked cytoplasmic accumulation of glial fibrillary acidic protein (gemistocytes) were also seen in the histopathological sections. An enlargement of the heterogeneous tumor in 6 $\mathrm{cm}$ diameter in the left-temporoparietal lobe was shifting the midline to the right on CT-images (Figure 2a).

The family history of the patient revealed an unknown femoral bone malignancy in his father. Although no significant extra- and intra-oral swellings were present, clinical examination showed localized decrease in left-maxillary vestibular depth from the canine to the second premolar region, relatively (Figure 3). Slight pain was present on palpation. There was no regional lymphadenopathy or any evidence suggests ocular involvement. While radiological examination with orthopantomography (OPG) showed resorption at the internal surface of the left maxillary sinus (Figure 4), maxillary occlusal radiograph revealed an enlarged left maxillary sinus with poorly defined margins (Figure 5). Left-maxillary sinus filled by a mass in $36 \times 53 \times 57 \mathrm{~mm}$ dimensions was also apparent in CT-images (Figure 2b). Invasion of the left-maxillary sinus mass into the adjacent tissues were remarkable with its extension to the anterolateral-, inferior-, medial-wall of the maxillary sinus, orbital cavity and pterygopalatinal fossa (T4). CT-images showed that the multiple gemistocytic astrocytomas (Figure 2a) were coexist with the left maxillary sinus mass (Figure $2 \mathrm{~b}$ ) on the preoperative CT-images (with contrast) obtained before any procedure was applied.

During an incision biopsy, destruction of the anterolateral wall of the left-maxillary sinus was observed. The tumor under the periost was in accord with the maxillary sinus mucosa, 
but continued with a soft, fragile, hyperplasic, reddish-purple lesion underneath. The lesion had tendency to bleeding easily and the hemorrhage occurred during biopsy was able to be controlled with haemostatic agents. Histopathological examination revealed it to be a well differentiated


Figure 1. Histopathological sections of gemistocytic astrocytoma. The sections are characterized with predominance of gemistocytes, which are neoplastic cells (1a (100xHE), 1b (200xHE)). Voluminous, eosinophilic cytoplasm, eccentric nuclei, plump cell process and marked cytoplasmic accumulation of glial fibrillary acidic protein (gemistocytes) are seen in the histopathological sections.

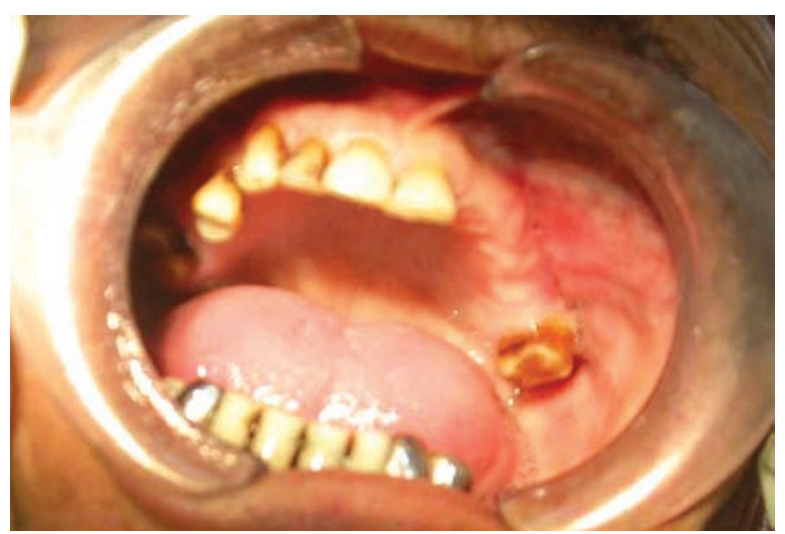

Figure 3. Relatively localized decrease in left-maxillary vestibular depth from canine to the second premolar region.
(Grade I) fibrosarcoma (Figure 6a, b, c, d). HEstained histopathological sections of fibrosarcoma showed spindle-cells crossing each other under the pseudo-stratified ciliary's epithelium at the surface (Figure 6a (40xHE)) and a mitosis (Figure 6b, arrow, (400xHE)). Vimentin was seen diffuse
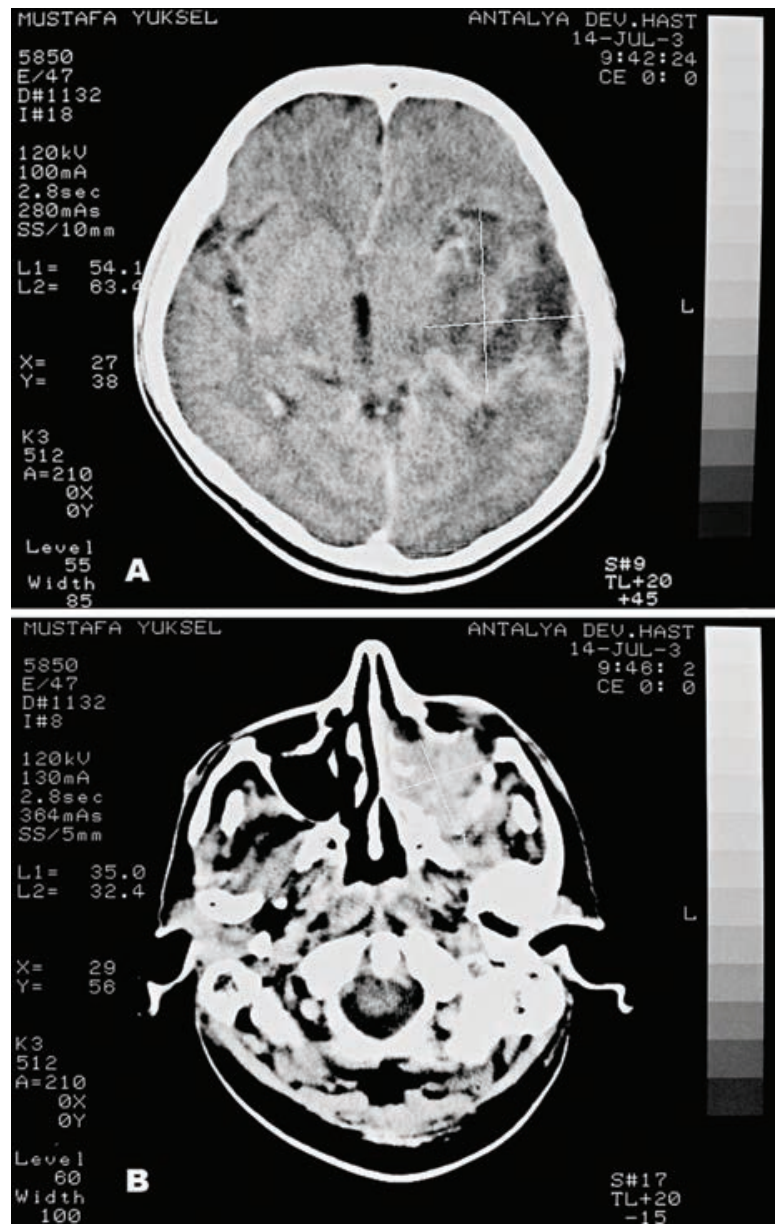

Figure 2. CT-images belong to brain and maxillary sinus. Ctimages taken before any procedure applied illustrating two separate tumors in the brain (2a) and left-maxillary sinus (2b). Note the enhancing heterogeneous tumor at the lefttemporoparietal lobe shifting the midline to the right (2a); and invasion of the tumor (T4) in the left-maxillary sinus into the adjacent tissues (2b).

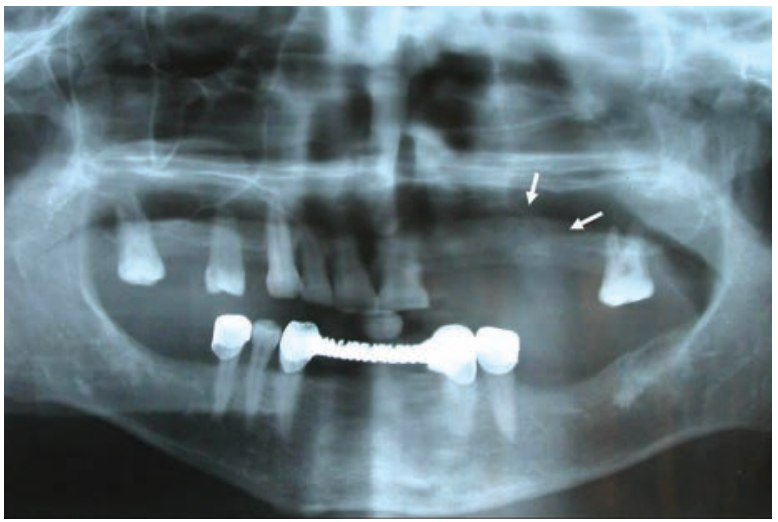

Figure 4. Orthopantomography shows resorption at the internal surface of the left-maxillary sinus. 
positive in tumor tissue (Figure $6 c(100 x$ Vimentin)) whereas SMA was seen negative in tumor tissue and positive in the vessel wall (Figure $6 \mathrm{~d}$ (100xSMA)).

According to treatment plan, having partial maxillectomy was performed (Figure 7a, b, c), the floor of the orbit was reconstructed with prolene mesh (Figure 7d) and split-skin grafts were applied to the excision site (Figure 7e). An immediate obturator with $1 \mathrm{~cm}$ extension into the excision side was stabilized with adams retention clasp to support the defect area and splint-skin graft on the day of surgery (Figure $7 f, g, h)$. Greater retention and stability was

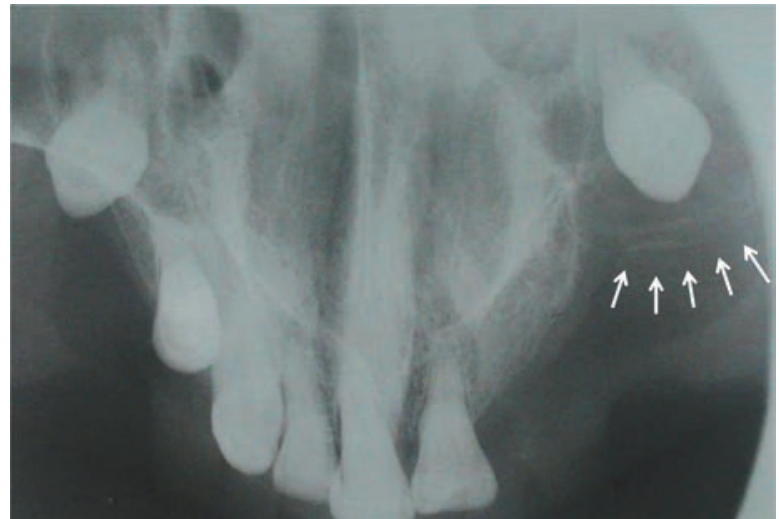

Figure 5. Maxillary occlusal radiograph illustrates an enlarged left maxillary sinus with poorly defined margins.
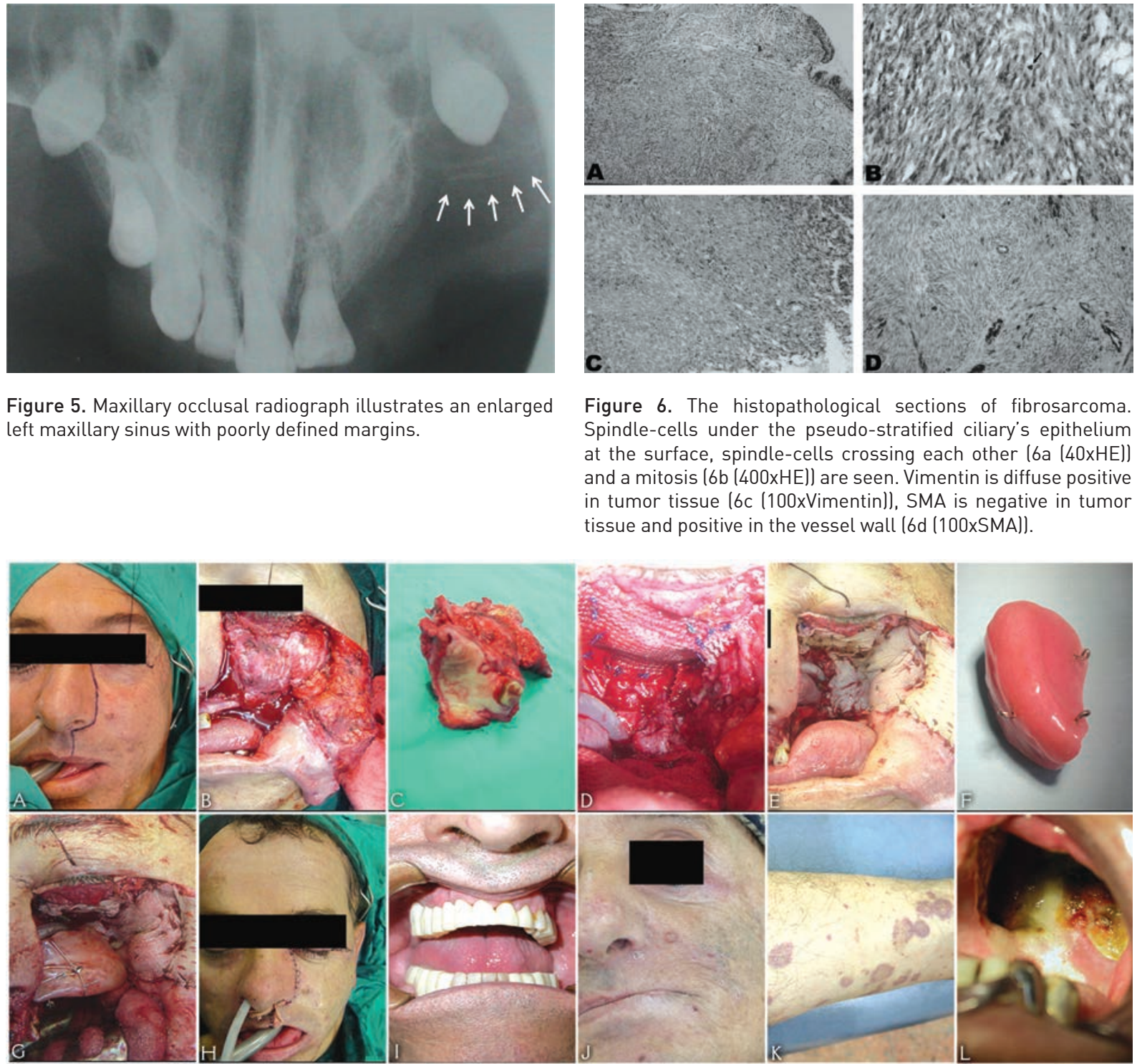

Figure 6. The histopathological sections of fibrosarcoma. Spindle-cells under the pseudo-stratified ciliary's epithelium at the surface, spindle-cells crossing each other (6a (40xHE)) and a mitosis (6b (400xHE)) are seen. Vimentin is diffuse positive in tumor tissue (6c (100xVimentin)), SMA is negative in tumor tissue and positive in the vessel wall (6d (100xSMA)).

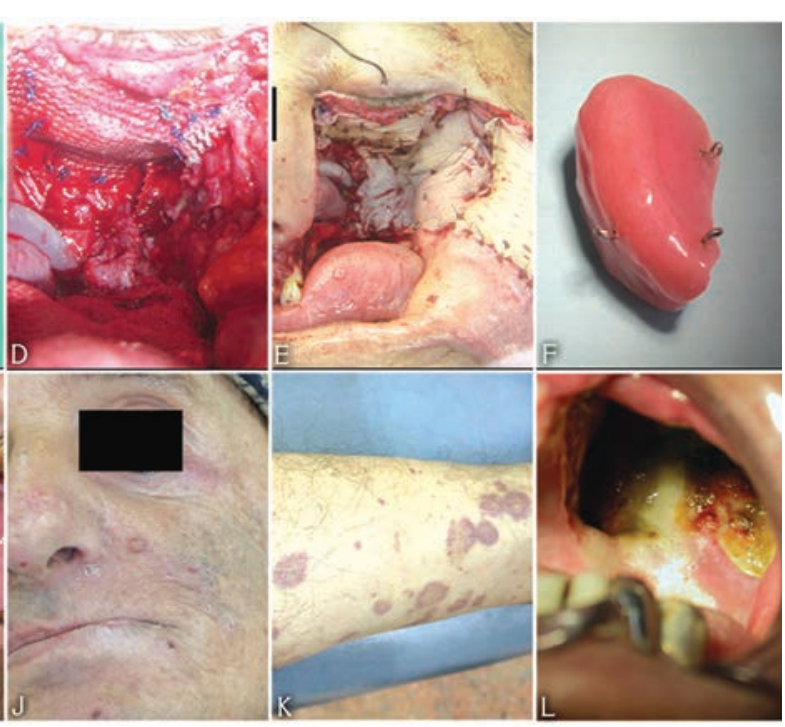

Figure 7. Clinical and surgical photographs of the patient. An incision line for partial maxillectomy (7a); exposed anterior wall of the maxillary sinus with fibrosarcoma (7b); excised tumor (7c); reconstruction of the floor of the orbit with prolen mesh (7d); application of full thickness skin graft (7e); intermit splint (7f) and its replacement for temporary reconstruction (7g); repositioning of the flap (7h); final reconstruction of the maxilla with an obturator (7i); leukocytoclastic vasculitis occurred on the face (7j) and leg (7k); skin graft lesions occurred (7I). 
distant metastases of the brain tumor at the time of maxillary sinus fibrosarcoma operation. An immediate postoperative course was uneventful. Patient received adjuvant radiotherapy with a total of 5000cGy in 5-weeks (200 cGy / day; 5 days / a week) with Cobalt-60 Penatherapy (Picker ATC C-9) three-months after the operation. Acute reactions to radiotherapy were mild and relatively self-limiting. Among these reactions, generalized skin lesions distributed to whole body at the thirdweek of radiotherapy was prominent. However the lesions had been already subsided with residual pigmentation spontaneously, since the patient presented to our clinic two-weeks after the occurrence of the lesions (Figure 7j, k). The skin graft also underwent changes in appearance and had distressing smell during this period (Figure 7l). Therefore, it was revised under general anesthesia and an incision biopsy was obtained for the possibility of recurrence. The specimen was interpreted as inflammatory granulation tissue. There was clear response to oral etodolac (400 mg; 3x1) with significant resolution of the lesion without any recurrence. Biopsies were also performed for the skin lesions and subsequently interpreted as leukocytoclastic vasculitis (Figure 8), which was characterized with perivascular infiltration of granulocytes (PMN), karrhyorhexis of their nuclei (leukocytoclasia) and damage of the vessel wall, coupled with compromise of the lumen and extravasations of erythrocytes within the dermis (100xHE).

Patient had no local recurrences at the primary sites; however, had distant metastases to lung, spinal and femoral bones two-years after the first operation as detected with scintigraphy. Patient rejected further chemotherapy and was followed on regular calls for another 6-month until died following increased intracranial edema induced paralysis.

\section{DISCUSSION}

We reported a case, who developed synchronous maxillary sinus fibrosarcoma and gemistocytic astrocytoma of brain. Synchronous malignancies are rare occurrences and, in general, their pathogenesis is unknown. In general, the possible mechanisms for synchronous malignancies may range from heredity, age, a genetic link between the cancers, immunological-, and environmental- state to simply coincidence. Up to $44 \%$ of paranasal sinus malignancies are attributed to occupational exposures, including nickel, chromium, isopropyl oils, volatile hydrocarbons, and organic fibers that are found in the wood, shoe, and textile industries. ${ }^{12}$ In addition, human papillomavirus can be a cofactor. ${ }^{12}$ Specific associations found include squamous cell carcinoma in nickel workers and adenocarcinoma in workers exposed to hardwood dust and leather tanning. ${ }^{12}$ Since the presented case is the first, it is difficult to identify etiology of both tumors. However, the presence of cancer in his family and his occupation may suggest either genetic tendency or occupational exposure as causative factors. No other etiologies or risk factors were identified.

Early diagnosis seems to be the most important factor in the management of paranasal sinus malignancies, since the primary cause of death is failure to control local disease. However, in accordance with our case (T4 stage), malignant tumors of the paranasal sinuses are often found to be greatly advanced at the primary site by the time diagnosis is established ( $75 \%$ patients). ${ }^{12}$ In that sense, the relative lack of specific symptoms indicating that malignancy is present or lack of awareness and cautious on routine radiographic findings seems to be reasons behind such misinterpretation and delay in diagnosis. Key symptoms of malignancy are cranial neuropathies and proptosis. However, these are uncommon at initial presentation and suggest advanced disease. ${ }^{12}$ Sign and symptoms of maxillary sinus carcinoma could be classified as nasal, ocular, facial, auditory and oral. ${ }^{12}$ Nasal findings occur approximately $50 \%$ of patients and consist of obstruction, discharge, stuffiness, congestion, epistaxis, and extension into the nasal cavity. Ocular findings are seen in up to $25 \%$ of patients and arise from upward extension into the orbit, where unilateral tearing, diplopia, fullness of lids, pain, and exophthalmos are seen. Facial signs comprise infraorbital nerve hypestesia, cheek swelling, pain, and facial asymmetry. Audiotory complaints consist of hearing loss secondary to serous otitis media due to nasopharyngeal extension. Finally, oral presentations arise in $25-35 \%$ of patients and include pain involving the maxillary dentition, trismus, palatal and alveolar ridge fullness, and frank erosion into the oral 
cavity. Multiple unilaterally missing left maxillary teeth in the presented case indicates to unrealized maxillary sinus fibrosarcoma development, and related pain as a causative factor for previous extractions. It should also be emphasized that although advanced imaging techniques often provide important additional diagnostic information which can help guide patient management, conventional radiographs le.g. OPG, maxillary occlusal radiograph or periapical radiographs) may also be valuable for the initial diagnosis of a lesion in the maxillary sinus as seen in the presented case. Since the success in the management and prognosis in cancer patients is highly associated with early diagnosis, suspicion leading to more detailed investigations should be performed when patients do not respond to routine dental therapy.

The survival of patients with fibrosarcoma is intermediate (60-70\%) in head and neck region, ${ }^{2}$ poor $130-50 \%$ for 5 -year) in maxillary sinus, ${ }^{13}$ and worst in gemistocytic astrocytoma $116 \%$ for 5-years; $0 \%$ for 10 -years). ${ }^{7}$ The overall survival of our patient was 2.5-years. The possible factors influenced the survival negatively were male sex, increasing age, higher TNM staging, site of the tumor (worse in the maxilla) for fibrosarcoma; ${ }^{11}$ and age, tumor grade and histological type (Grade II - poorly differentiated) for gemistocytic astrocytoma. ${ }^{5,7}$

It was difficult to apply a standard treatment protocol for the presented case due to absence of the similar presentation in the literature. Therefore, understanding of the clinical

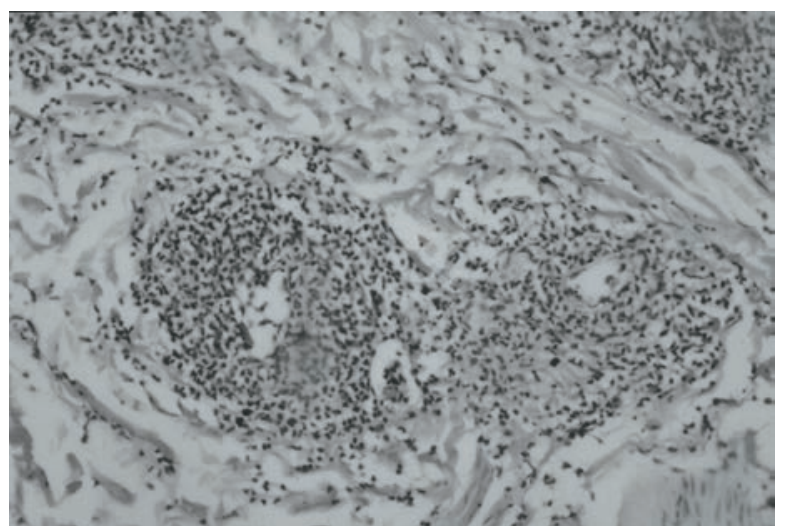

Figure 8. Histopathological section of leukocytoclastic vasculitis. Section is characterized with perivascular infiltration of granulocytes (PMN), karrhyorhexis of their nuclei (leukocytoclasia) and damage of the vessel wall, coupled with compromise of the lumen and extravasations of erythrocytes within the dermis (100xHE). behaviors of the tumors in response to therapy was also vague. The best treatment protocol for such patients awaits further documentations and investigations. However, in relevance with maxillofacial surgery, wide surgical resection with clear margins is important for a favorable survival, since fibrosarcoma is more of a locally destructive problem (recurrence rate: 30 $66.7 \%)^{14}$ than a metastatic problem (distant metastases rate: $\sim 20 \%)^{14}$ Furthermore, it is known that postoperative radiotherapy is capable to augment surgical 5 -year cure rate by $10-15 \% .{ }^{14}$ Therefore the combined modality of surgery and adjuvant radiotherapy, which is widely accepted to be the most effective combination, ${ }^{15,16}$ was used for the presented case. However, distant metastases and the survival period of this case may suggest that application of chemotherapy, which is interventional at present, as a third modality may have additional value for the management and longer survival of such cases. Our assumption seems to be supported with a study reported the beneficial effect of chemotherapy in combination with surgery or radiotherapy. ${ }^{11}$

Development of leukocytoclastic vasculitis, which is an autoimmune disease, during the course of radiotherapy, was another unusual observation in the presented case. Although, it could be discussed whether the precipitating factor for the leukocytoclastic vasculitis was radiotherapy or malignancies, ${ }^{17}$ it is known that leukocytoclastic vasculitis may also develop independent from any cancer. ${ }^{18}$ To our knowledge, the induction of leukocytoclastic vasculitis has not been reported in the context of radiotherapy, so far. Whether autoimmune phenomena occur after irradiation therapy is obscure and requires further investigations to explore. Whatsoever, such unusual presentation and clinical hallmarks of leukocytoclastic vasculitis lead we to emphasize that cancer patients will receive radiotherapy should be monitored for the potential development of leukocytoclastic vasculitis.

This report constitutes data in terms of clinical course, prognosis, treatment modality and survival of such synchronous malignant tumors, and open discussion on likelihood radiotherapyinduced leukocytoclastic vasculitis. 


\section{ACKNOWLEDGEMENT}

The authors thank to Suleyman Demirel University Research and Application Hospital for affording treatment expenses of the patient.

\section{REFERENCES}

1. Osguthorpe JD, Richardson M. Frontal sinus malignancies. Otolaryngol Clin North Am 2001;34:269-281.

2. Wanebo HJ, Kones RJ, MacFarlane JK, Eilber FR, Byers RM, Elias EG, et al. Head and neck sarcoma: report of the Head and Neck Sarcoma Registry Society of Head and Neck Surgeons Committee on Research. Head Neck 1992;14:1-7.

3. Figueiredo MT, Marques LA, Campos-Filho N. Soft-tissue sarcomas of the head and neck in adults and children: experience at a single institution with a review of literature. Review. Int J Cancer 1988;15:198-200.

4. Gorsky M, Epstein JB. Head and neck and intra-oral soft tissue sarcomas. Oral Oncol 1998;34:292-296.

5. Krouwer HG, Davis RL, Silver P, Prados M. Gemistocytic astrocytomas: a reappraisal. J Neurosurg 1991;74:399-406.

6. Kleihues P, Davis RL, Ohgaki H, Burger PC, Westphal MM, Cvenee WK. Diffuse astrocytoma. In: Kleihues P, Cavenee WK, editors. Pathology and genetics of tumors of the nervous system. Lyon: IARC Press 2000;22-26.

7. Okamoto Y, Di Patre PL, Burkhard C, Horstmann S, Jourde $B$, Fahey $M$, et al. Population-based study on incidence, survival rates, and genetic alterations of low-grade diffuse astrocytomas and oligodendrogliomas. Acta Neuropathol 2004;108:49-56.

8. Sanroman JF, Hoyo JRA, Diaz FJ, Gil-Diez L, Monje F, Naval L, Alamillos FJ, Rubio ADP. Sarcomas of the head and neck. Br J Oral Maxillofac Surg 1992;30:115-118.

9. Nagler RM, Malkin L, Ben-Arieh Y, Laufer D. Sarcoma of the maxillofacial region, follow-up of 25 cases. Anticancer Res 2000;20:3735-3742.

10. Pandey M, Thomas G, Mathew A, Abraham EK, Somanathan T, Ramadas K, type EM, Ahamed IM, Sebastain P, Nair MK. Sarcoma of the oral and maxillofacial soft tissue in adults. Eur J Surg Oncol 2000;26:145-148.

11. Yamaguchi S, Nagasawa $H$, Suzuki T, Fujii E, Iwaki $H$, Takagi $M$, et al. Sarcomas of the oral and maxillofacial region: a review of 32 cases in 25 years. Clin Oral Investig 2004;8:52-55.

12. Katzenmeyer K, Pou A. Neoplasms of the Nose and Paranasal Sinus. In: Dr.Quinn's Online Textbook of Otolaryngology. June 7, 2000.

13. Le QT, Fu KK, Kaplan M, Terris DJ, Fee WE, Goffinet DR. Treatment of maxillary sinus carcinoma: a comparison of the 1997 and 1977 American Joint Committee on cancer staging systems. Cancer 1999;86:1700-1711.
14. Ou SM. Soft tissue sarcoma in the head and neck-analysis of 87 patients. Zhonghua Zhong Li Za Zhi 1988;10:289-292.

15. Hayashi T, Nonaka S, Bandoh N, Kobayashi Y, Imada M, Harabuchi Y. Treatment outcome of maxillary sinus squamous cell carcinoma. Cancer 2001;92:1495-1503.

16. Eeles RA, Fisher $C, A^{\prime}$ Hern RP, Robinson M, Rhys-Evans $P$, Henk JM, Archer D, Harmer CL. Head and neck sarcomas, prognostic factors and implications for treatment. $\mathrm{Br} \mathrm{J}$ Cancer 1993;68:201-207.

17. Greer JM, Longley S, Edwards NL, Elfenbein GJ, Panush RS. Vasculitis associated with malignancy. Experience with 13 patients and literature review. Review. Medicine (Baltimore) 1988; 67: 220-230.

18. Hutson TE, Hoffman GS. Temporal concurrence of vasculitis and cancer: a report of 12 cases. Arthritis Care Res 2000;13:417-423. 